\title{
Drug Discovery through Nuclear Magnetic Resonance Spectroscopy: From Binding Epitopes and Ligand Screening to Conformational Analysis
}

\author{
Brian Cutting*
}

\begin{abstract}
Nuclear magnetic resonance (NMR) methods at the Institute of Molecular Pharmacy (IMP) provide crucial information in several fundamental themes in drug discovery. Assignments of complex carbohydrates are obtained to a high degree of completion and accuracy, thereby accelerating their identity. Conformational analysis of ligands in their unbound state reveals to what extent the steric considerations, essential for minimizing entropy losses upon binding, are optimized. Small libraries of compounds are screened for their binding to receptors. Promising ligands are analyzed in the presence of their receptor to determine the geometric arrangements in the bound state, as well as the functional groups responsible for the observed binding.
\end{abstract}

Keywords: Conformational analysis · Ligand binding · Nuclear magnetic resonance ·

Saturation transfer difference

\section{Introduction}

Magnetic resonance in drug discovery has become a vital technology in the search for promising drug candidates. The methods have witnessed explosive growth in their applicability to diverse areas in the pharmaceutical sciences. Recognition of the scientific contributions from magnetic resonance techniques is testified by the recent Nobel Prizes awarded in 2003 for medicine and 2002 for chemistry, for imaging and structural biology, respectively.

The importance of magnetic resonance imaging is easily recognized in several ar-

${ }^{*}$ Correspondence: Dr. B. Cutting Institute of Molecular Pharmacy

Department of Pharmaceutical Sciences

Pharmacenter

University of Base

Klingelbergstr 50

$\mathrm{CH}-4056$ Basel

Tel.: +41612671563

Fax: +416126715 51

E-Mail: brian.cutting@unibas.ch

www.pharma.unibas.ch/molpharm/index.htm/ eas of drug discovery and development. The technique provides a non-invasive visualization of pharmacokinetics, as well as target identification and validity [1]. In parallel to imaging methods, high-resolution liquid state NMR allows the characterization of the structure and dynamics of receptors with or without the ligand, and is therefore of fundamental importance to strategies employing structure-based drug design. The considerable information contained in NMR methods which observe the receptor has been acknowledged in a special issue of ChemBioChem [2]. In addition, significant progress has been achieved in the structural characterization of membrane-bound proteins by solid-state NMR [3][4]. These investigations promise to increase the number of targets amenable to structure-based drug discovery. The scope of this report is highly restricted to a description of a limited number of NMR techniques that are currently in use at the Institute of Molecular Pharmacy.

\section{Resonance Assignment and Conformational Analysis}

NMR assignments of oligosaccharides are complicated by the dense cluster of signals resonating in the vicinity of 3.1-4.3 ppm [5-7]. To understand the influence that different functional groups of the ligand contribute to binding, the correspondence between the individual components in the dense cluster, and the part of the ligand from which they originate, must be confidently assigned. In protein NMR spectroscopy, incorporation of magnetic heteronuclei, such as ${ }^{13} \mathrm{C},{ }^{15} \mathrm{~N}$ and possibly ${ }^{2} \mathrm{H}$, is a pre-requisite for their assignment [8][9]. Dispersion of the large number of protein resonances onto a second or third dimension is necessary, since, for these types of molecules, on the order of 1,000 resonances are observed in the ${ }^{1} \mathrm{H}$ spectrum. The repertoire of established three-dimensional experiments used for protein NMR spectroscopy, such as HSQC-TOCSY, suffers from the low natural abundance of ${ }^{13} \mathrm{C}$. Fortunately, selective experiments circumvent the demand for very high-resolution or multi-dimensional experiments when studying oligosaccharides.

Selective one-dimensional analogs of numerous three-dimensional experiments are readily adapted to the study of unlabelled carbohydrates [10]. Because of the restriction of spectral content inherent to selective experiments, the one-dimensional spectra often exhibit highly resolved peaks. However, the restriction of the information to a particular component of the molecule implies that, to achieve a full assignment, multiple selective experiments must be performed.

An example of the use of selective experiments at our institute is in the assignment of a trisaccharide ligand [11] of the myelin-associated glycoprotein (MAG) [12]. Selec- 


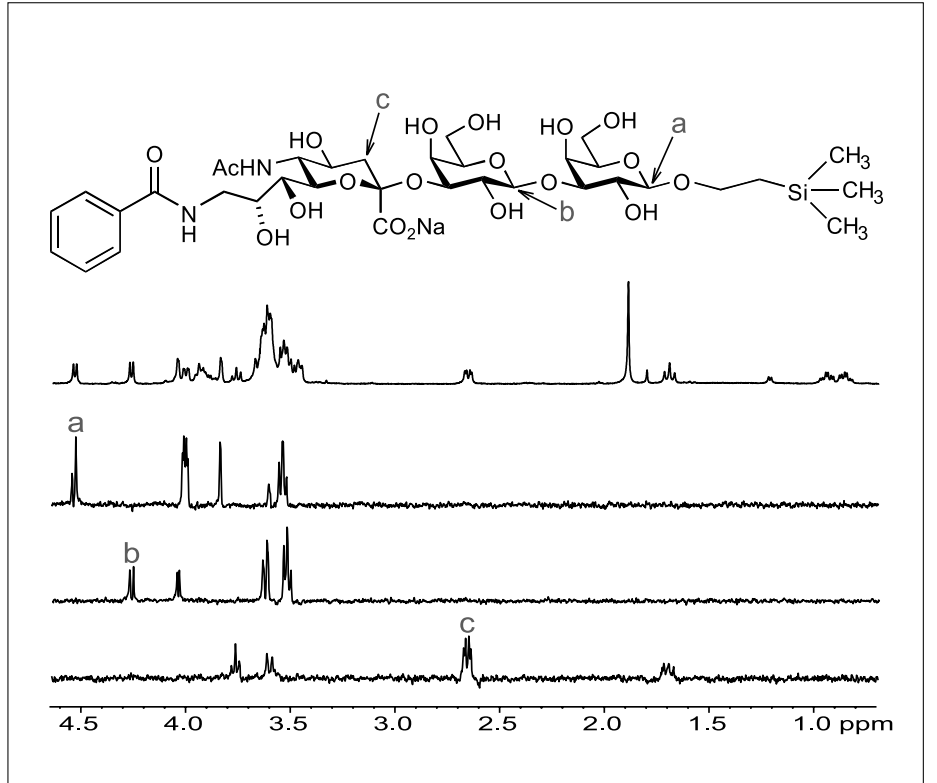

Fig. 1. Spin-system identification of a trisaccharide MAG ligand through selective TOCSY experiments. The letters, ' $a$ ', 'b' and 'c' refer to different hydrogen atoms used as a sources of magnetization. The upper spectrum shows the region of interest in a non-selective experiment. The spectra below show the result of a 100 ms TOCSY mixing time following the selective excitation of hydrogen ' $a$ ', 'b' or 'c', respectively.

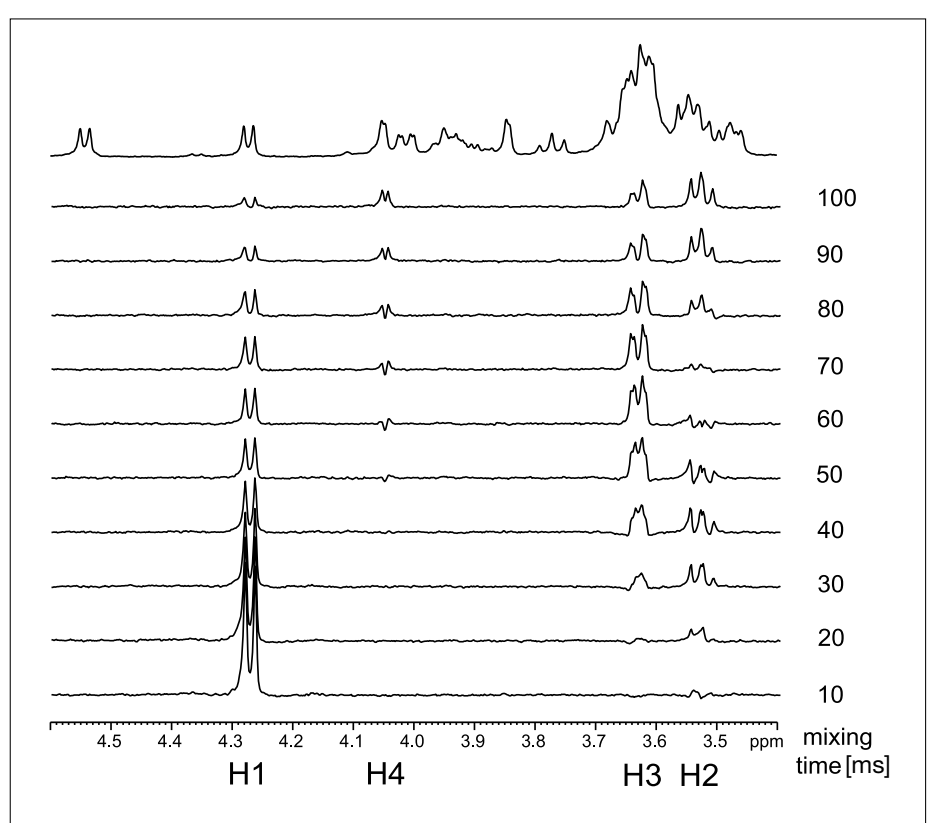

Fig. 2. The kinetics of the TOCSY transfer can be used for assignment. Spectra recorded at short mixing times (below) transfer magnetization successively to scalar coupled partners at longer mixing times (above). The assignments shown were initially obtained with traditional NMR methods (COSY, HSQC, and HMBC). tive TOCSY experiments [13] were used to identify the scalar-coupled spin-systems for each residue of the trisaccharide (Fig. 1). It is noteworthy that the non-selective two-dimensional TOCSY [14], would not have resulted in such a straightforward identification of scalar-coupled networks because of spectral overlap. To isolate the spin-systems in an oliogosaccharide, one isolated resonance in each spin-system is required for the source of TOCSY transfer [15]. Fortunately, for carbohydrates of moderate size, isolated anomeric protons are nearly always present, due to the paucity of other signals in this region.

An additional advantage of choosing the anomeric proton as the source of TOCSY magnetization is that, during shorter mixing times, the flow of the transfer proceeds along successive protons in the network [16]. Specifically, magnetization is transferred from the source proton, 'i', to proton ' $i+1$ '. By contrast, if the source of TOCSY transfer was not chosen to be at the terminus of the spincoupled chain, the scalar coupled partners preceding and following the chosen proton would complicate the kinetics of the TOCSY transfer. The initial time dependence of the TOCSY transfer can be used to rapidly assign resonances. A typical result applied to the above-mentioned MAG ligand is shown in Fig. 2.

To increase the affinity of $\mathrm{sLe}^{\mathrm{x}}$ mimics for E-Selectin [17], it is important to minimize entropy losses by a pre-organization of the molecule into a bioactive conformation [18]. Pre-organization is achieved through the introduction of steric substituents which increase the population of $\mathrm{sLe}^{\mathrm{x}}$ mimics with a conformation of the fucose residue optimally stacked underneath the galactose residue in its most favorable binding mode to E-Selectin. It is often possible to estimate the extent of stacking of the residues by an analysis restricted to measuring the distance between the Fuc-H5 and Gal-H2 hydrogens. This has been performed in our institute on $\mathrm{sLe}^{\mathrm{x}}$ through selective one-dimensional ROESY measurements [19], and is presented in
Fig. 3. The selective one-dimensional experiments are attractive due to the high signal-tonoise ratio, spectral simplicity, and resolution achieved. In the middle of the ROESY period, two non-selective pi pulses were applied and the frequency of the spin-lock was switched from high to low field to prevent TOCSY artifacts (JS-ROESY) [20].

Signals from the oligosaccharides are quantitated either by non-selective two-di- a)

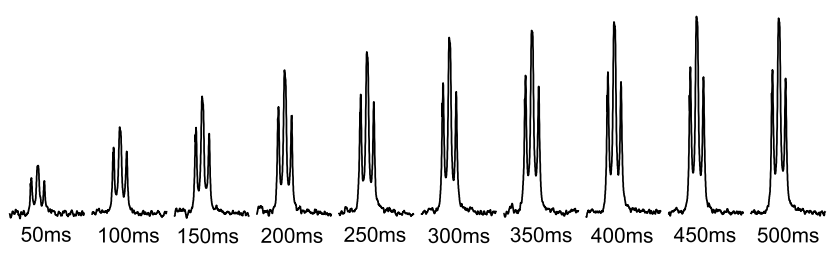

b)

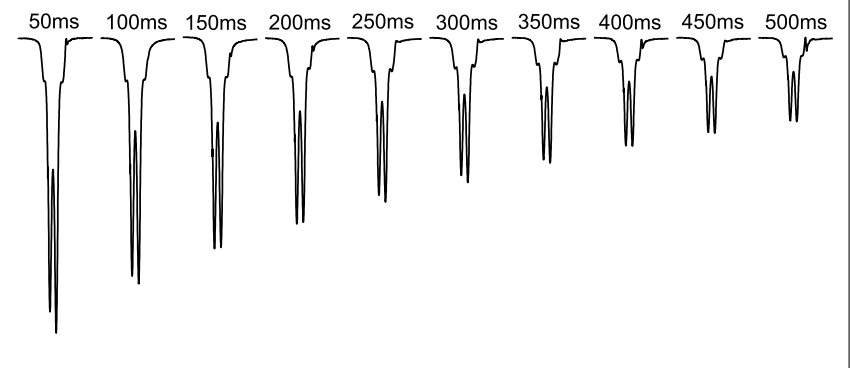

Fig. 3. Selective ROESY experiments measuring the transfer of magnetization from the Fuc-H5 to Gal-H2 in a sLex mimic. Analysis of the time-dependence of the transfer of magnetization to the target peak (a) from the source peak (b) provides a distance constraint. The average internuclear distance was determined to be approximately $2.6 \AA \AA$. 
mensional experiments, or by direct application of selective pulses. In some cases, the signal of interest resonates in the previously mentioned area of 3.1 to $4.3 \mathrm{ppm}$, and is thus often buried underneath other signals. Nonetheless, these signals may often be isolated, without recourse to ${ }^{13} \mathrm{C}$ enrichment, provided they are scalar coupled to another resonance that is in an isolated part of the spectrum. Selective transfer of magnetization from the isolated resonance to the overlapped resonance proceeds by the doubly-selective homonuclear Hartmann-Hahn method (dsHOHAHA) [21]. Experimentally this transfer is realized through the simultaneous application of low amplitude spin-lock fields applied at the frequencies of both resonances, where the fields are applied for a duration equal to the inverse of the scalar coupling between them. If the resulting spectrum is not of sufficient purity following the dsHOHAHA, a selective gradient echo may be appended to the sequence. The result of the dsHOHAHA scheme coupled to a selective gradient echo, applied to a mimic of $\mathrm{sLe}^{\mathrm{x}}$, is shown in Fig. 4.

As has been described above, pre-organization of the ligand into a conformation that is similar to that observed in the bound state will minimize affinity losses due to entropy. Pre-organization, however, presupposes that the bound conformation of the ligand is known. At the IMP, this is determined through transferred Overhauser measurements [22] from the receptor to the ligand (Fig. 5). The method takes advantage of the fact that small ligands exhibit minimal cross-relaxation rates when in the free form, which are greatly enhanced when bound to a large receptor.

\section{Ligand Screening}

Several excellent review articles have already been published that describe in considerable detail the potential of NMR to identify small ligands binding to receptors [23][24]. Methods based on observation of the ligand resonances take advantage of the quicker diffusion of the smaller ligand. This property is manifested in the NMR spectrum by changes in the relaxation rates, or linewidth, of the ligand through binding. Upon binding of the ligand to the receptor, the transverse and selective longitudinal time a)

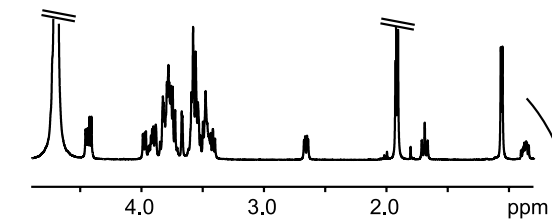

b)

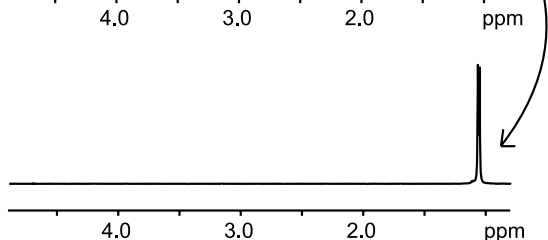

c)

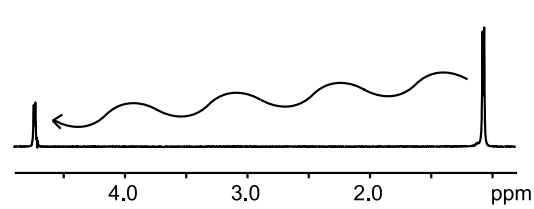

d)

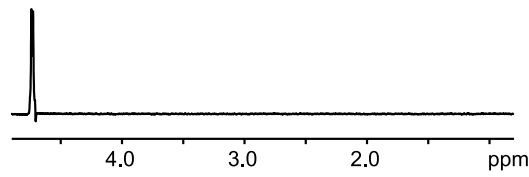

e)

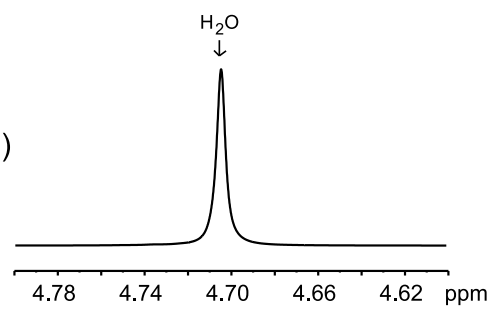

f)

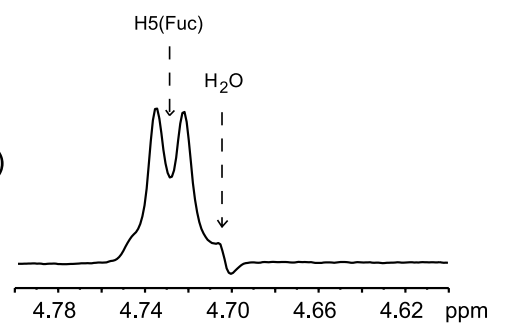

Fig. 4. The dsHOHAHA scheme is an effective method to selectively excite signals in the case of overlap. The signal of interest, the Fuc- $\mathrm{H} 5$ of a $\mathrm{sLe}^{\mathrm{x}}$ mimic is buried underneath the intense water resonance. The Fuc- $\mathrm{H} 6$ is relatively isolated, and may be selectively excited by traditional shaped pulses (b). Magnetization is passed from Fuc-H6 to Fuc-H5 by the dsHOHAHA method (c), which is isolated by appending a selective gradient echo (d). Expansion of the region of interest demonstrates that the water resonance in the non-selective spectrum (e) obscures the Fuc-H5 resonance, the latter is isolated with the method described (f). The procedure would be successful even if the resonance maxima were identical, provided one of the signals has a scalar coupled partner that could be isolated.

Fig. 5. Transferred Overhauser (trNOESY) experiments are used to determine the conformation of ligands when bound to receptors. The spectrum of the ligand alone, corticosterone (Sigma-Aldrich) at $2 \mathrm{mM}$, has no observable cross-peaks (a). When mixed with the receptor, $100 \mathrm{MM}$ human serum albumin (Sigma-Aldrich), cross peaks are clearly seen (b). The two spectra were measured with identical spectrometer conditions and plotted using the same contour values. 
constants are shorter. Additionally, the Overhauser effect is more efficient with larger molecules, or equivalently, small ligands binding to large receptors. The more negative Overhauser enhancement allows experiments such as transferred NOESY (trNOESY) and the saturation transfer difference (STD) to be readily observed for small ligands binding to large receptors.

At the IMP, several methods are used to evaluate the affinity a small ligand has for a receptor. To increase the reliability of the evaluation, different experiments are performed to find a consistent agreement. An example of the use of transverse relaxation to demonstrate NMR-observed binding is shown in Fig. 6. For the nonbinding compound, the slight difference between the relaxation with and without the protein is due to the overlap of quickly relaxing protein resonances and slowly relaxing resonances of the non-binding ligand.

In addition to increases in auto-relaxation rates to observe binding, intermolecular cross-relaxation between the receptor and the ligand is a complementary diagnostic method. At the IMP, the STD experiment has proven to be an effective means to observe complexation between ligands and receptors. The experiment has been thoroughly described elsewhere [25], here however, a short explanation is provided. The STD measures the intermolecular NOE from the receptor to the ligand, provided the latter binds to the former. The receptor is saturated through the application of radio-frequency pulses in a part of the spectrum where the receptor, but not the test compound, has resonances. Magnetization is transferred throughout the receptor due to spin diffusion. The resonances of the ligand that receive the largest magnetization transfer are nearest to a large density of magnetization of the receptor, and hence likely to be important for binding. An example of the STD method, to discriminate between non-binding and binding compounds, is shown in Fig. 7.

\section{Binding Epitopes}

The STD experiment, in addition to being a screening method for ligand binding, is also used to determine the relative contribution of different functional groups of the ligand to the observed binding. Quantitation of the relative amount of magnetization transferred to different hydrogen nuclei in the ligand describes the contacts made to the receptor, and is referred to as STD epitope mapping [26][27]. An example of this method applied in the IMP to a ligand of the MAG protein [28] is shown in Fig. 8.

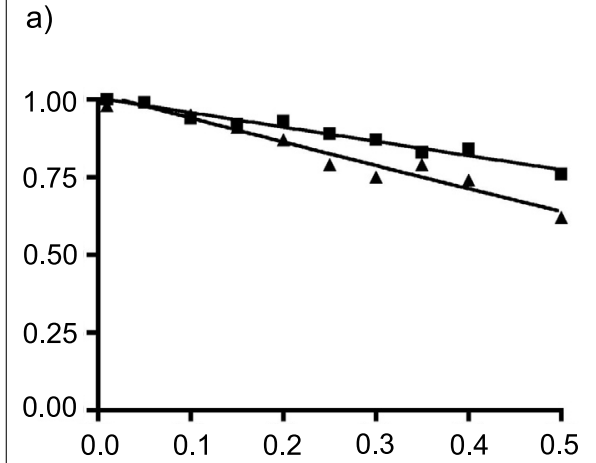

b)

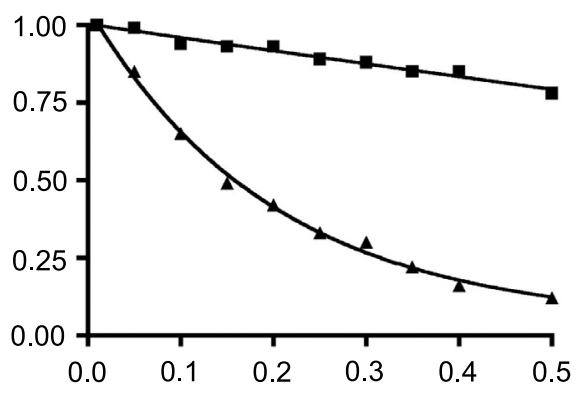

Fig. 6. Transverse relaxation is used as a method to screen for binding ligands. (a) $\beta$-D-glucose shows a minor difference in relaxation in the absence $(\mathbf{\square})$ or presence of human serum albumin in a 20:1 ratio $(\boldsymbol{\Lambda})$. Binding is clearly observed with corticosterone (b). Corticosterone relaxes slowly in isolation,(口) but much faster with a $20: 1$ ratio of human serum albumin $(\mathbf{\Lambda})$.

a)

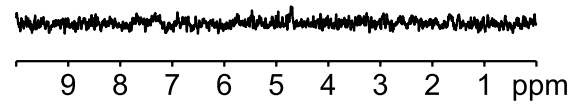

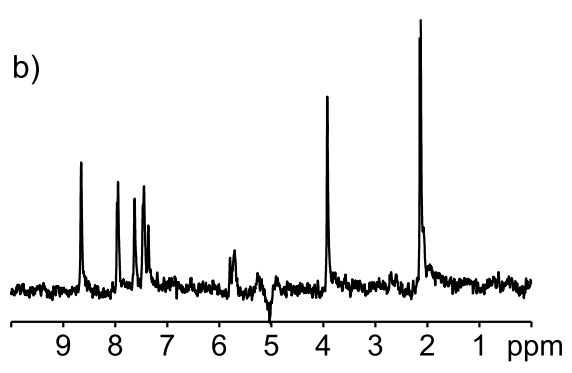

Fig. 7. The STD experiment is used to discriminate between binding and non-binding ligands. $\beta$ D-glucose at $1 \mathrm{mM}$ is not seen to bind to $20 \mu \mathrm{M}$ human serum albumin (a), whereas binding of corticosterone at $1 \mathrm{mM}$ to $20 \mu \mathrm{M}$ human serum albumin is clearly observed (b).

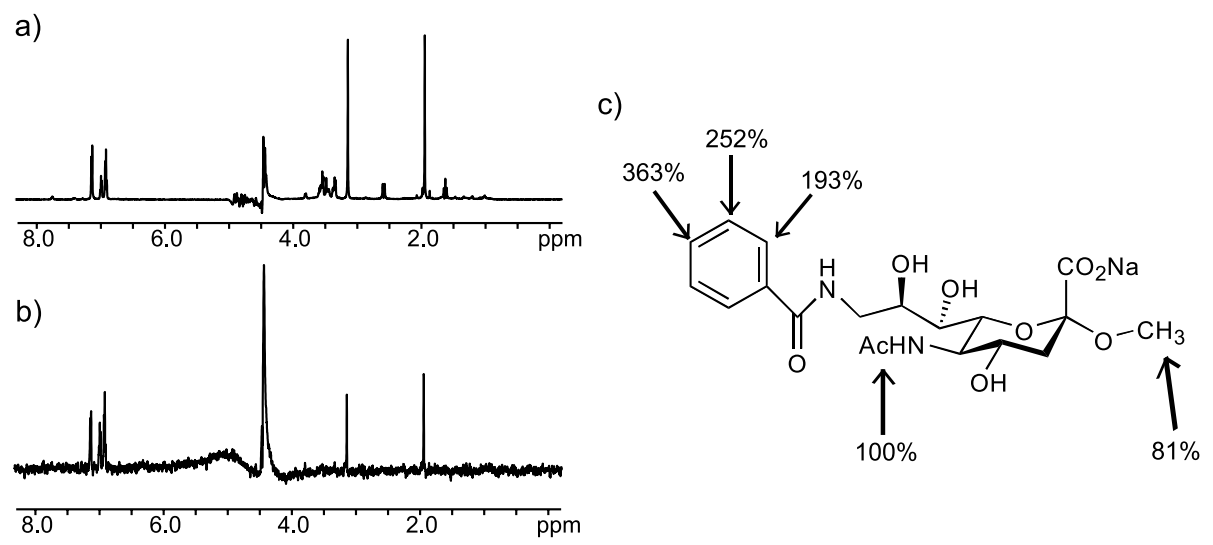

Fig. 8. The binding epitopes of ligands are measured with the STD experiment. The epitope is measured by integrating a peak in the STD spectrum (b) with the corresponding peak in the reference spectrum (a). The most significant STD values, with respect to the $\mathrm{N}$-acetate signal that has been assigned a value of $100 \%$, are mapped onto the ligand (c).

\section{Conclusions}

Nuclear magnetic resonance methods at the Institute of Molecular Pharmacy provide a crucial interface between biology, chemistry, molecular modeling, and other biophysical techniques. Selective TOCSY allows the rapid identification of spin-sys- tems in complex carbohydrates. The kinetic measurement of the TOCSY transfer results in an expeditious assignment of residues within each spin-system. Ligand conformations in the unbound state are measured with ROESY experiments. When spectral overlap is troublesome, doubly-selective Hartmann-Hahn experiments greatly en- 
hance the number of signals amenable to measurement without the need for isotopic enrichment. Ligand binding is analyzed with several complementary experiments to increase the reliability of hit determination. Transferred NOESY and STD measurements function both as a means to validate binding and a method to determine the bound state conformation and contacts responsible for binding.

\section{Acknowledgements}

The author is grateful to Sachin Shelke and Dr. Ganpan Gao for the synthesis of the MAG ligands. Dr. Heiko Gathje and Prof. Soerge Kelm from the University of Bremen for the MAG protein sample. Dr. Christian Mueller and Bea Wagner for preparing the $\mathrm{sLe}^{\mathrm{x}}$ mimic. Prof. Beat Ernst is gratefully acknowledged for his encouragement and support of all aspects of the projects described in this article. Financial support for these projects was generously provided by the Swiss National Science Foundation and the Volkswagen Foundation.

Received: December 22, 2005

[1] N. Beckmann, D. Laurent, B. Tigani, R. Panizzutti, M. Rudin, Drug Disc. Tod. 2004, 9(1), 35.

[2] H. Schwalbe, H.U. Stilz, H. Kessler, ChemBioChem 2005, 6, 1475.

[3] A. Watts, Nature Rev. Drug. Discov. 2005, 4, 555 .

[4] O.C. Andronesi, S. Becker, K. Seidel, H. Heise, H.S. Young, M. Baldus, J. Am. Chem. Soc. 2005, 127, 12965.

[5] J.O. Duss, C.H. Gotfredsen, K. Bock, Chem. Rev. 2000, 100, 4589.

[6] 'NMR Spectroscopy of Glycoconjugates', Eds. J. Jimenez-Barbero, T. Peters, WileyVCH, Weinheim, 2003.

[7] W.A. Bubb, Concepts Magn. Reson. 2003, $19,1$.

[8] J. Cavanagh, W.J. Fairbrother, A.G. Palmer, N.J. Skelton, 'Protein NMR Spectroscopy: Principles and Practice', Academic Press, New York, 1996.
[9] M. Sattler, J. Schleucher, C. Griesinger, Prog. Nuc. Mag. Res. Spect. 1999, 34, 93.

[10] H. Kessler, S. Mronga, G. Gemmecker, Mag. Reson. Chem. 1991, 29, 527.

[11] G. Gao, O. Schwardt, T. Visekruna, S. Rabbani, B. Ernst, Chimia 2004, 4, 215.

[12] S. Kelm, R. Brossmer, R. Isecke, H.J. Gross, K. Strenge, R. Schauer, Eur. J. Biochem. 1998, 255, 663.

[13] D.G. Davis, A. Bax, J. Am. Chem. Soc. 1985, 107, 7197.

[14] L. Braunschweiler, R.R. Ernst, J. Magn. Reson. 1983, 53, 521.

[15] C. Roumestand, C. Delay, J.A. Gavin, D. Canet, Mag. Reson. Chem. 1999, 37, 451.

[16] R. Marx, S.J. Glaser, J. Magn. Reson. 2003, 164,338

[17] B. Ernst, Z. Dragic, S. Marti, C. Mueller, B. Wagner, W. Jahnke, J.L. Magnani, K.E. Norman, R. Oehrlein, T. Peters, H.C. Kolb, Chimia 2001, 4, 268.

[18] G. Thoma, J.L. Magnani, J.T. Patton, B. Ernst, W. Jahnke, Angew. Chem. Int. Ed. 2001, 40, 1941.

[19] A.A. Bothner-By, R.L. Stephens, J. Lee, C.D. Warren, R.W. Jeanloz, J. Am. Chem. Soc. 1984, 106, 811.

[20] J. Schleucher, J. Quant, S.J. Glaser, C. Griesinger, J. Mag. Reson. 1995, 112, 144.

[21] B. Boulat, R. Konrat, I. Burghardt, G. Bodenhausen, J. Am. Chem. Soc. 1992, 114, 5412.

[22] P. Balaram, A.A. Bothner-By, J. Dadok, $J$. Am. Chem. Soc. 1972, 94, 4015.

[23] B.J. Stockman, C. Dalvit, Prog. Nuc. Mag. Res. Spect. 2002, 41, 187.

[24] J.W. Peng, J. Moore, N. Abdul-Manan, Prog. Nuc. Mag. Res. Spect. 2004, 44, 225.

[25] B. Meyer, P. Peters, Angew. Chem. Int. Ed. 2003, $42,864$.

[26] M. Mayer, B. Meyer, Angew. Chem. Int. Ed. 1999, 38, 1784.

[27] M. Mayer, B. Meyer, J. Am. Chem. Soc. 2001, 123, 6108.

[28] S. Kelm, R. Brossmer, 'Neuraminic Acid Derivatives for use as Siglec Inhibitors' Patent Number: CA2451051, 2003. 